\title{
Estudo sobre a audição em idosos e associação com sintomatologia depressiva
}

\author{
Amanda Kunzler Etcheverria*, Ivana Oliveira Prediger*, Magda Aline Bauer ${ }^{* * *}$, \\ Andréa Kruger Gonçalves "***, Andréa Ortiz Corrêa "****, Mirian Sana Barreto Biggoweit" ${ }^{* * * *}$, \\ Luciane Raquel Steiner Zanotto"****, Adriane Ribeiro Teixeira ${ }^{*+* * * *}$
}

\section{Resumo}

A perda auditiva representa uma das principais causas de isolamento social nos idosos e pode levar à depressão. O objetivo deste estudo foi verificar a existência de associação entre a presença e o grau de perda auditiva e sintomatologia depressiva em um grupo de idosos, bem como analisar a associação entre essas duas variáveis e a influência da idade e do sexo. Foram avaliados indivíduos idosos, por meio de audiometria tonal liminar, com pesquisa de limiares tonais por via aérea e via óssea, para verificação da presença, tipo e grau de perda auditiva. A avaliação também incluiu a aplicação da escala de depressão geriátrica (GDS) para observar a presença de sintomatologia depressiva. Fizeram parte da amostra 96 idosos, sendo $74(77,1 \%)$ do sexo feminino, com idades

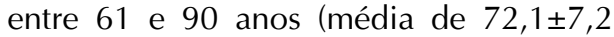

anos). Verificou-se presença de perda auditiva em $64(66,7 \%)$ idosos, com predomínio de perda auditiva leve e moderada. Quanto à sintomatologia depressiva, essa foi observada em $22(22,9 \%)$ idosos. Não foi observada associação entre a presença e o grau de perda auditiva e sintomatologia depressiva. Houve associação entre a perda auditiva e a idade. Concluiu-se que a maior parte dos idosos apresentou perda auditiva de grau leve a moderado. A sintomatologia depressiva foi detectada em um número menor de indivíduos e não houve associação entre perda auditiva e sintomatologia depressiva. A idade exerceu influência sobre a presença de perda auditiva. Não houve associação entre o sexo, a perda auditiva e a sintomatologia depressiva.

Palavras-chave: Perda Auditiva. Depressão. Envelhecimento.

* Fonoaudióloga (UFRGS), Especializanda em Audiologia (UFRGS).E-mail: amanda_kunzler@hotmail.com

** Fonoaudióloga (UFRGS). E-mail: ivanasiloli@hotmail.com

**** Fonoaudióloga do Curso de Fonoaudiologia (UFRGS), Mestre em Distúrbios da Comunicação Humana (UFSM). E-mail: magdafono@gmail.com

**** Educadora Física, Doutora em Psicologia (USP), Professora do Departamento de Educação Física (UFRGS) e Coordenadora do Curso de Especialização em Envelhecimento e Qualidade de Vida (UFRGS). E-mail: andreakg@terra.com.br

****** Fonoaudiólogas do Serviço de Fonoaudiologia do Grupo Hospitalar Conceição, Porto Alegre (RS).

******* Fonoaudióloga, Especialista em Audiologia (CFFA) e Gerontologia (SBGG), Mestre em Distúrbios da Comunicação Humana (UFSM) e Doutora em Gerontologia Biomédica (PUCRS). Professora do Departamento de Saúde e Comunicação Humana (UFRGS). E-mail: adriane.teixeira@gmail.com

$\rightarrow$ http://dx.doi.org/10.5335/rbceh.2012.4049

Recebido em: 15/06/2014. Aprovado em: 02/09/2014 


\section{Introdução}

Envelhecer não é mais um privilégio de poucos indivíduos, mesmo que a melhora dos parâmetros de saúde das populações observada no século XX esteja longe de se distribuir de forma equitativa nos diferentes países e contextos socioeconômicos. $\mathrm{O}$ crescimento da população idosa é um fenômeno mundial, mesmo nos países mais pobres como o Brasil, no qual essas modificações estão ocorrendo de forma radical e acelerada, indicando que, em 2020, o país ocupará a sexta posição em número de idosos (VERAS, 2009).

$\mathrm{O}$ fato de a expectativa de vida ter aumentado não significa que as condições de vida para o idoso também tenham melhorado (SILVA et al, 2007). Envelhecer no Brasil ainda significa um aumento no número de doenças crônicas (DANIELEWICZ; BARBOSA; DEL DUCA, 2014). Das alterações sensoriais sofridas por esses indivíduos, a diminuição da acuidade auditiva representa uma das principais causas de isolamento social, configurando-se como a alteração que produz maior impacto na comunicação e na vida psicossocial, podendo levar também à ansiedade, à depressão e à privação das atividades de vida diária do idoso (NOBREGA, CÂMARA, BORGES, 2008, KIM; CHUNG, 2013).

A perda auditiva causada pelo processo de envelhecimento é denominada presbiacusia. Geralmente, ela é bilateral, simétrica e progressiva, sendo o resultado de um somatório de fatores extrínsecos e intrínsecos (ROSENHALL, 2003). Mesmo tendo sido realizados vários estudos, tentando associar a presença e o grau de perda auditiva à exposição ao ruído, fatores hereditários, ototoxicidade, dieta, metabolismo, aterosclerose e hipertensão arterial, a causa ainda permanece incerta (GARDNER; SLENKOVICH, 2006).

A perda da audição é a terceira condição crônica mais prevalente entre os idosos, sendo menor apenas do que a hipertensão arterial e a artrite, com uma ligeira tendência para os homens apresentarem maior prevalência, nas diferentes faixas etárias (GRECO; RUSSO, 2006, VALETE-ROSALINO; ROZENFELD, 2005, KIDD; BAO, 2012). Por sua elevada prevalência, a presbiacusia pode ser considerada um problema de saúde pública (CRUICKSHANKS et al., 2003, McCORMACK; FORTNUM, 2013).

A presbiacusia provoca distúrbios psicológicos, sociais e emocionais, com redução da vida social e aumento dos problemas de relacionamento com familiares, amigos e no trabalho. Esses distúrbios afetam de forma negativa as atividades de vida diária do idoso e sua mobilidade (MONZANI et al, 2008, NACHTEGAAL et al, 2009, MALHOTRA; CHAN; OSTBYE, 2010). Dentre as alterações que podem estar associadas à perda auditiva, está a depressão.

Assim, o objetivo deste estudo é verificar a presença e o grau de perda auditiva e sintomatologia depressiva em um grupo de idosos, bem como analisar a associação entre essas duas variáveis e a influência da faixa etária e do sexo. 


\section{Material e métodos}

Este estudo teve delineamento transversal, observacional e descritivo. A amostra foi composta por indivíduos com idade igual ou superior a 60 anos, de ambos os sexos. Dentre os critérios de inclusão, estavam: histórico negativo de doenças e/ou internações por problemas neurológicos e/ou psiquiátricos, declarar-se apto e interessado em participar da pesquisa e assinar o termo de consentimento livre e esclarecido (TCLE), ausência de cera obstrutiva no meato acústico externo e a realização completa das avaliações selecionadas para a pesquisa. Optou-se por excluir os idosos com distúrbios neurológicos e/ou psiquiátricos já diagnosticados, para evitar a inclusão de idosos em tratamento para tais doenças, o que poderia influenciar nas respostas ao instrumento GDS e, consequentemente, nos resultados finais.

Os participantes da pesquisa frequentavam um projeto de extensão na universidade ou eram atendidos no Setor de Fonoaudiologia de um hospital da cidade de Porto Alegre. Os idosos foram abordados enquanto aguardavam o início das atividades do projeto de extensão ou $o$ atendimento fonoaudiológico. Foram convidados a participar da pesquisa pelos responsáveis pela coleta de dados. Inicialmente, era feita a explicação dos objetivos da pesquisa, dos procedimentos a serem realizados e dos benefícios e riscos a que estariam expostos, bem como do caráter voluntário da participação.

Os indivíduos que aceitaram participar voluntariamente da pesquisa foram conduzidos a uma sala silenciosa, para a assinatura do Termo de Consentimento Livre e Esclarecido. A seguir, responderam à escala de depressão geriátrica (GDS) - versão abreviada (SHEIKH; YESAVAGE, 1986), de forma individual, em forma de entrevista. A GDS é um instrumento com 15 questões para o rastreio de sintomatologia depressiva. Na sequência, foram encaminhados para a cabina acústica, para a realização de audiometria tonal liminar. Foram pesquisados os limiares tonais por via aérea (de $250 \mathrm{~Hz}$ a $8000 \mathrm{~Hz}$ ) e por via óssea (de $500 \mathrm{~Hz}$ a $4000 \mathrm{~Hz}$ ), utilizando-se audiômetro da marca Interacoustics, modelo AC30.

A presença e o grau de perda auditiva foram determinados utilizando-se a classificação da Organização Mundial da Saúde (OMS, 1997), que consiste no cálculo da média dos limiares obtidos nas frequências de $500 \mathrm{~Hz}, 1000 \mathrm{~Hz}, 2000 \mathrm{~Hz}$ e $4000 \mathrm{~Hz}$. Médias de até 25dBNA indicam que os limiares auditivos são normais; valores entre $26 \mathrm{dBNA}$ e $40 \mathrm{dBNA}$ configuram perdas auditivas de grau leve; entre 41dBNA e 60dBNA de grau moderado; entre 61dBNA e 80dBNA grau severo e superiores a $81 \mathrm{dBNA}$ de grau profundo.

De acordo com as normas de interpretação da GDS, a cada resposta que evidenciasse tendência depressiva era atribuído um ponto. A avaliação foi feita da seguinte forma: menos de cinco pontos - ausência de sintomatologia depressiva; entre 5 e 10 pontos - sintomatologia depressiva leve a moderada; 11 pontos ou mais, sintomatologia depressiva grave (FIGUEIREDO et al., 2002). 
O protocolo de pesquisa foi aplicado pelos fonoaudiólogos e pelos acadêmicos do curso de Fonoaudiologia participantes da pesquisa. No caso da aplicação por acadêmicos, sempre houve supervisão de profissional responsável. Para uniformizar a aplicação da GDS e da audiometria tonal liminar, houve uma capacitação prévia, para que os procedimentos fossem aplicados de maneira uniforme e com redução de viés de pesquisa.

O projeto foi aprovado pelo Comitê de Ética em Pesquisa da instituição (número 2010035) e pelo Comitê de Ética do Grupo Hospitalar (número 145211). Todos os participantes assinaram um Termo de Consentimento Livre e Esclarecido, baseado na Resolução 196/96 do Ministério da Saúde.

Os resultados das variáveis nominais foram expressos por meio de análises de frequência e os resultados das variáveis contínuas por meio de média \pm desvio padrão. Para verificar a relação do grau de perda auditiva com sexo, faixa etária, lado da orelha e o grau de depressão foi utilizado o teste qui-quadrado de acordo com as suposições do teste. Para verificar a associação entre a média dos limiares auditivos com o sexo e o lado da orelha, foi utilizado o teste $T$ student para variáveis independentes, e, para verificar a associação entre as médias dos limiares auditivos com as categorias de faixa etária, frequência e grau de depressão, foi utilizado o teste One Way Anova, quando necessário o teste de comparações múltiplas. Para verificar o grau de correlação entre a idade e a escala de depressão, foi utilizada a análise de correlação de Pearson e para verificar a normalidade dos dados foi utilizado o teste Kolmogorov-Smirnov. Para todas as análises, foi considerado significativo um valor de $\mathrm{p}<0,05$.

\section{Resultados}

Foram incluídos na amostra 96 indivíduos, de ambos os sexos. As idades variaram de 61 a 90 anos, sendo a média

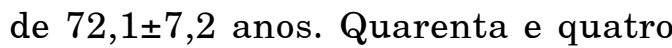
indivíduos $(45,8 \%)$ tinham entre 60 e 70 anos, $40(41,7 \%)$ entre 71 e 80 anos, e $12(12,5 \%)$ entre 81 e 90 anos. A maior parte dos pacientes era do sexo feminino $(77,1 \%)$.

$\mathrm{Na}$ Tabela 1, estão descritos os valores máximo, mínimo e médio dos limiares audiométricos encontrados, bem como o desvio padrão em cada frequência. Em ambas as orelhas, observa-se um aumento nas médias dos limiares auditivos em função do aumento da frequência. 
Tabela 1: Limiares audiométricos estratificados por orelha e por frequência

\begin{tabular}{|c|c|c|c|}
\hline Frequência & Valor mínimo & Valor máximo & Média \pm desvio padrão \\
\hline \multicolumn{4}{|l|}{ Orelha direita } \\
\hline $250 \mathrm{~Hz}$ & 5 & 110 & $29,53 \pm 16,56$ \\
\hline $500 \mathrm{~Hz}$ & 5 & 115 & $30 \pm 17,52$ \\
\hline $1000 \mathrm{~Hz}$ & 5 & 115 & $31,67 \pm 18,40$ \\
\hline $2000 \mathrm{~Hz}$ & 5 & 115 & $38,23 \pm 19,69$ \\
\hline $3000 \mathrm{~Hz}$ & 5 & 115 & $41,88 \pm 22,78$ \\
\hline $4000 \mathrm{~Hz}$ & 5 & 115 & $45,21 \pm 24,72$ \\
\hline $6000 \mathrm{~Hz}$ & 5 & 115 & $52,71 \pm 26,36$ \\
\hline \multirow[t]{2}{*}{$8000 \mathrm{~Hz}$} & 5 & 120 & $54,58 \pm 26,78$ \\
\hline & 10 & 100 & $36,66 \pm 18,56$ \\
\hline \multicolumn{4}{|l|}{ Orelha esquerda } \\
\hline $250 \mathrm{~Hz}$ & 0 & 110 & $30,89 \pm 18,76$ \\
\hline $500 \mathrm{~Hz}$ & 5 & 115 & $31,35 \pm 19,35$ \\
\hline $1000 \mathrm{~Hz}$ & 0 & 95 & $31,41 \pm 20,43$ \\
\hline $2000 \mathrm{~Hz}$ & 0 & 100 & $37,24 \pm 20,76$ \\
\hline $3000 \mathrm{~Hz}$ & 0 & 100 & $41,93 \pm 22,61$ \\
\hline $4000 \mathrm{~Hz}$ & -5 & 115 & $44,95 \pm 24,46$ \\
\hline $6000 \mathrm{~Hz}$ & 5 & 120 & $56,04 \pm 24,85$ \\
\hline $8000 \mathrm{~Hz}$ & 0 & 105 & $54,43 \pm 24,45$ \\
\hline
\end{tabular}

Fonte: dos autores.

Na Figura 1, são apresentados os limiares auditivos médios estratificados por frequência e faixa etária, separados por orelha. 
Figura 1: Média dos limiares auditivos estratificados por frequência e faixa etária

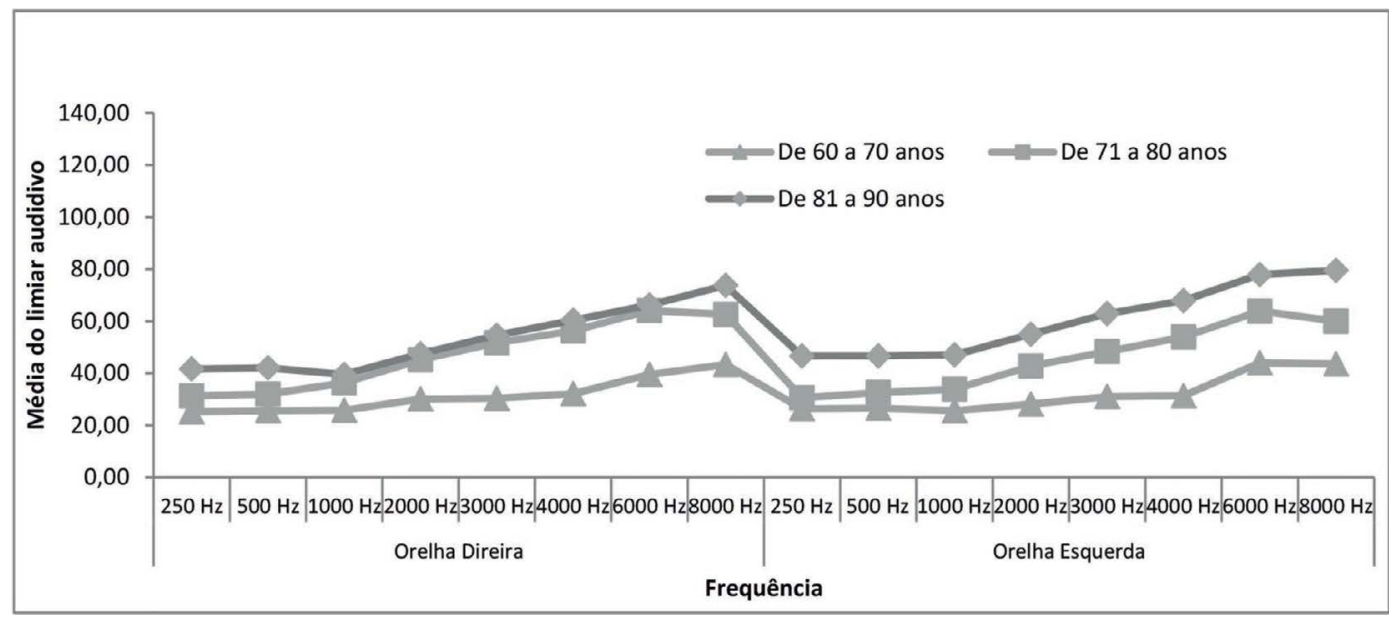

Fonte: dos autores.

Na Figura 2, são apresentados os dados relacionados ao grau de perda auditiva, por orelha. Verifica-se que predominou a presença de indivíduos com limiares auditivos normais, perda auditiva leve e perda auditiva moderada.

Figura 2: Classificação dos limiares auditivos por orelha

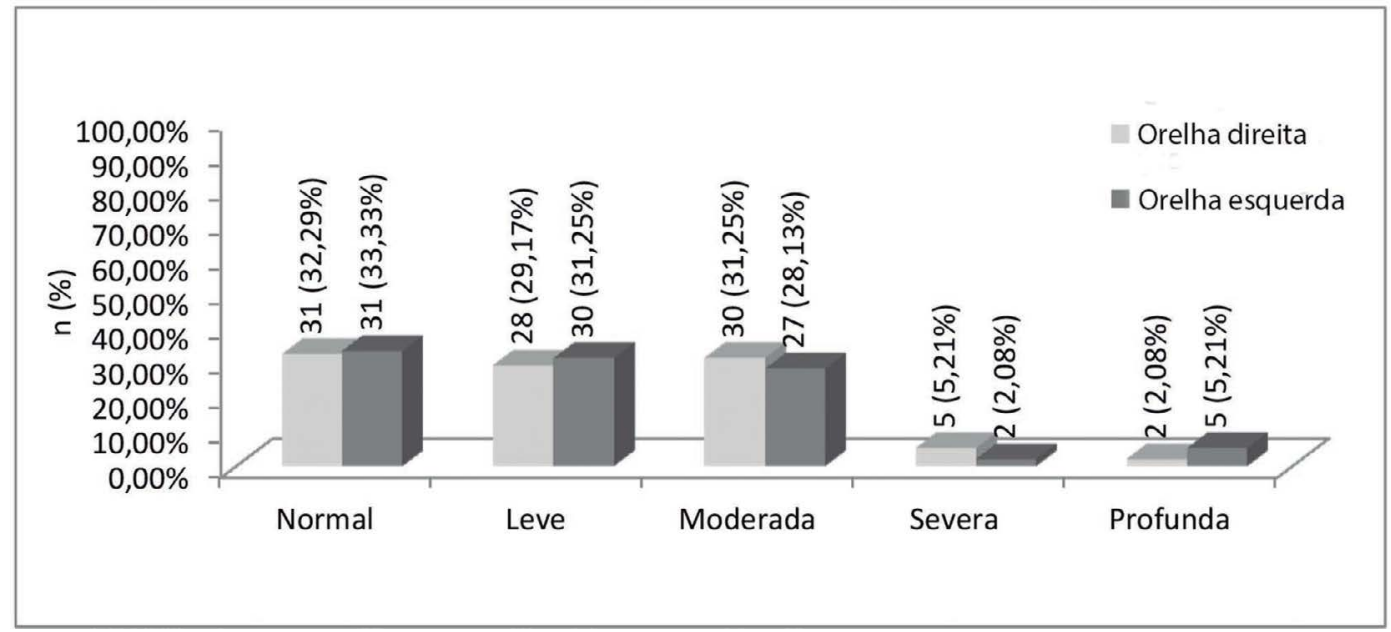

Fonte: dos autores.

Na Tabela 2, são apresentados os dados referentes à sintomatologia depressiva na amostra estudada. Não foi observada correlação significativa entre a pontuação da GDS, o sexo e a faixa etária. 
Tabela 2: Estatística descritiva dos resultados da GDS

\begin{tabular}{|c|c|c|c|c|}
\hline & \\
\hline & & Valor mínimo & Valor máximo & Média \pm desvio padrão \\
\hline \multicolumn{2}{|c|}{ GDS - pontuação geral } & 0 & 14 & $3,86 \pm 2,29$ \\
\hline \multirow{6}{*}{$\begin{array}{l}\text { Pontuação } \\
\text { GDS }\end{array}$} & Sexo & & & \\
\hline & Masculino & 0 & 14 & $4,36 \pm 2,57$ \\
\hline & Feminino & 0 & 11 & $3,74 \pm 3,07$ \\
\hline & 60 a 70 anos & 7 & 11 & $4,17 \pm 2,80$ \\
\hline & 71 a 80 anos & 0 & 14 & $4,2 \pm 3,56$ \\
\hline & 81 a 90 anos & 1 & 6 & $4 \pm 1,70$ \\
\hline
\end{tabular}

Nota: Correlação de Pearson: $r=0,01$ e $p=0,92$.

Fonte: dos autores.

Na Tabela 3, são apresentados os dados da análise da associação entre presença/ausência de perda auditiva e as variáveis sexo, faixa etária e sintomatologia depressiva. Observou-se associação somente entre o sexo, a faixa etária e a presença de perda auditiva. Não se constatou associação entre perda auditiva e sintomatologia depressiva, embora o maior percentual de idosos com sintomatologia depressiva apresente audição alterada.

Tabela 3: Associação entre a presença/ausência de perda auditiva e as demais variáveis de estudo

\begin{tabular}{|c|c|c|c|}
\hline & \multirow{2}{*}{\multicolumn{2}{|c|}{ Audição }} & \\
\hline & & & \multirow[b]{2}{*}{$\mathrm{p}$} \\
\hline Variáveis & $\begin{array}{c}\text { Normal } \\
n=32\end{array}$ & $\begin{array}{c}\text { Alterada } \\
\mathrm{n}=64\end{array}$ & \\
\hline Sexo & & & $0,02^{*}$ \\
\hline Masculino & $4(12,50 \%)$ & $18(28,13 \%)$ & \\
\hline Feminino & $28(87,50 \%)$ & $46(71,88 \%)$ & \\
\hline Faixa etária & & & $0,02^{*}$ \\
\hline De 60 a 70 & $25(78,13 \%)$ & $19(29,69 \%)$ & \\
\hline De 71 a 80 & $6(18,75 \%)$ & $34(53,13 \%)$ & \\
\hline De 81 a 90 & $1(3,13 \%)$ & $11(17,19 \%)$ & \\
\hline Depressão & & & 0,70 \\
\hline Ausência & $26(81,25 \%)$ & $48(75 \%)$ & \\
\hline Presença & $6(18,75 \%)$ & $16(25 \%)$ & \\
\hline
\end{tabular}

Fonte: dos autores. 
Na Figura 3 são apresentados os resultados da análise da associação entre o grau de perda auditiva da melhor orelha e o grau de sintomatologia depressiva.
Constatou-se que não houve associação estatisticamente significativa entre estas variáveis $(\mathrm{p}=0,28)$.

Figura 3: Associação entre os limiares auditivos na melhor orelha e o grau de sintomatologia depressiva

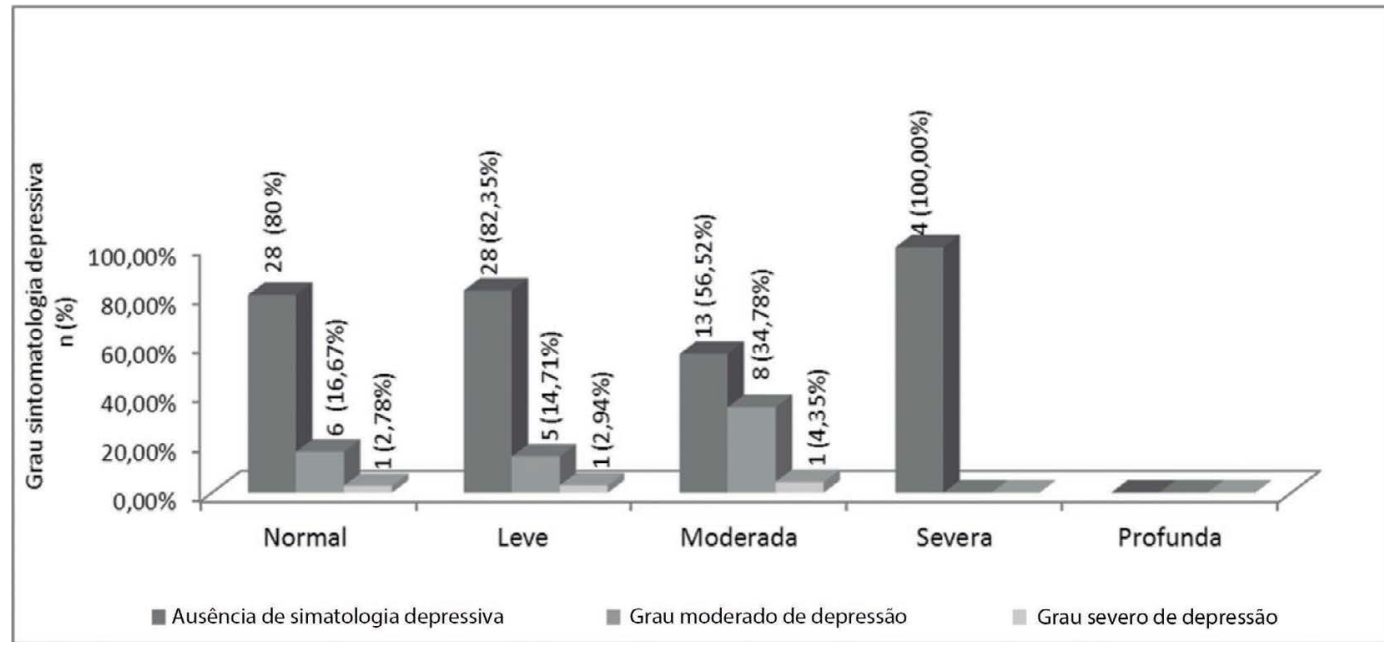

\section{Discussão}

A deficiência auditiva é um dos problemas decorrentes do processo de envelhecimento e pode gerar problemas de comunicação. Essa perda de audição é progressiva e compromete, principalmente, as frequências altas. A instalação gradual faz com que o idoso demore a perceber que está com perda auditiva, mas pode comprometer, desde o início, a inteligibilidade da fala nas situações sociais. Dessa forma, o convívio social torna-se cada vez mais difícil e pode originar isolamento social (GRECO; RUSSO, 2006, KIM; CHUNG, 2013).

A análise dos dados evidenciou que a amostra foi composta, em sua maioria, por indivíduos do sexo feminino. Esse fato pode ser explicado pela feminilização do envelhecimento, fato já constatado anteriormente e confirmado pelos dados do censo 2010 (IBGE, 2012). Além disso, como parte da amostra foi composta por idosos pertencentes a um grupo de terceira idade, esse fator certamente influenciou os resultados. É evidente que a maior parte dos idosos que frequentam tais atividades é do sexo feminino, o que já foi descrito por outros autores (LEITE et al., 2006, ZAITUNE et al., 2010).

A análise dos limiares auditivos por frequência evidenciou que, quanto menores as frequências avaliadas, melhores os limiares auditivos. $\mathrm{O}$ aumento frequencial foi acompanhado de aumento de limiares auditivos. Esse resultado pode 
ser explicado pelo tipo de perda auditiva encontrado em indivíduos idosos. A característica da presbiacusia é o acometimento bilateral, simétrico e audiograma, com configuração descendente, ou seja, com maiores limiares auditivos em frequências altas (SAMELLI et al., 2011, TENÓRIO et al., 2011), corroborando o que foi obtido no estudo.

Quando consideradas as quatro faixas etárias estabelecidas no estudo, observou-se que há um aumento nas médias dos limiares auditivos, de acordo com o aumento da frequência e da idade. Esse dado era esperado pelos pesquisadores. Em idosos com 70 anos ou mais, não foram observados limiares auditivos normais, indicando que a prevalência de perda auditiva aumenta progressiva e proporcionalmente ao aumento da idade (NÓBREGA; CÂMARA; BORGES, 2008, BARALDI, ALMEIDA; BORGES, 2007, BESS; WILLIAMS; LICHTENSTEIN, 2001, MENESES et al. 2010, BEVILACQUA et al. 2013).

Com relação à perda auditiva, a análise dos dados coletados evidenciou que a maior parte dos idosos apresentou perda auditiva de grau leve ou moderado, o que corrobora estudos anteriores (BILTON et al., 1997, BÉRIA et al., 2007, MATTOS; VERAS, 2007, AMARAL; SENA, 2004, PEDALINI et al., 1997, TEIXEIRA et al., 2010).

Quanto à sintomatologia depressiva, verificou-se que 23 idosos (23,9\%) apresentaram sintomas depressivos de grau leve a profundo. Esse número é levemente inferior ao descrito na literatura especializada, a qual relata que aproximadamente $30,0 \%$ dos idosos não institucionalizados apresentam sintomas de depressão (MACIEL; GUERRA, 2006, FARIA; BARRETO, PASSOS, 2008). É importante destacar que muitos indivíduos da amostra são participantes do grupo de extensão da universidade, onde realizam atividades físicas e de lazer. Pesquisas sugerem que a prática de exercícios físicos pode proporcionar aos indivíduos a diminuição do estresse e da ansiedade, bem como pode aliviar os sintomas físicos e emocionais em pessoas deprimidas (FARIA; BARRETO; PASSOS, 2008, DUNN et al., 2005, BLUMENTHAL et al., 1999, DESLANDES, 2013, MINGHELLI et al., 2013). Esse fator pode ter influenciado os resultados obtidos, pois a prática de atividades sociais, de lazer e de esportes por parte dos idosos avaliados provavelmente está provocando efeitos benéficos no que se refere à ausência de sintomatologia depressiva.

Não houve associação entre a faixa etária e o grau de sintomatologia depressiva apresentada pelos idosos $(p=0,93)$, o que corrobora os achados do estudo de Faria, Barretoe Passos (2008). Assim, a idade, por si só, não aumentaria o risco de depressão. Estudos epidemiológicos sugerem que os efeitos da idade, no caso da sintomatologia depressiva, podem ser atribuídos aos problemas de saúde e às incapacidades frequentes nos idosos e não ao envelhecimento em si (COSTA et al., 2003). A análise dos dados evidenciou que não houve associação entre sexo e presença de sintomatologia depressiva, o que difere de estudos anteriores (COELHO et al., 2013, FERREIRA; TAVARES, 2013). 
Quando analisada a relação entre presença/ausência de perda auditiva e presença/ausência de sintomatologia depressiva, constatou-se que não houve associação significativa. Esse dado difere de estudos anteriores, que demonstram a presença dessa associação (TEIXEIRA et al., 2010, MILLÁN-CALENTI et al., 2011, LI et al., 2014).

A análise entre o grau de perda auditiva e de sintomatologia depressiva também não evidenciou associação estatisticamente significativa, contrariando a hipótese deste estudo. Considerando que a perda auditiva provoca dificuldade de compreensão e, por consequência, dificuldades de comunicação, originando isolamento social e familiar, acreditava-se que os idosos com maior perda auditiva apresentariam maior pontuação na GDS. Essa associação havia sido descrita em trabalhos anteriores, mesmo que com relações fracas ou menos consistentes (CHOU; CHI, 2004, McDONALL, 2009).

Esses resultados talvez possam ser explicados pelo número de indivíduos com limiares auditivos normais ou com perda auditiva leve na melhor orelha. De acordo com a OMS (1997), perdas auditivas leves não são incapacitantes, ou seja, não privam o indivíduo da vida em sociedade. Assim, os sujeitos com perda auditiva avaliados nesta pesquisa podem ainda não estar experimentando os efeitos da privação auditiva. Além disso, a prática de atividades variadas fora do ambiente do lar pode estar beneficiando tais sujeitos, auxiliando para que esses não apresentem sintomatologia depressiva.
Assim, na amostra estudada, foi constatada a presença de perda auditiva e depressão, mas não houve associação entre tais variáveis. Acredita-se, contudo, que os profissionais que atuam com pacientes geriátricos devem estar cientes de que muitos idosos apresentam tais distúrbios, de forma isolada ou conjunta, e que a avaliação especializada, bem como o tratamento específico, devem ser adotados assim que o diagnóstico for concluído.

Acredita-se que novos estudos deverão ser feitos, abordando, mais especificamente, idosos do sexo masculino. Como descrito anteriormente, a amostra deste estudo foi selecionada por conveniência, tendo como base idosos que aguardavam atendimento fonoaudiológico ou que participavam de projetos de extensão. Em tais locais, é muito maior a presença de mulheres, o que pode ser considerado uma limitação do estudo, uma vez que as mulheres buscam mais atendimento para os problemas de saúde e participam maciçamente de grupos de convivência.

\section{Conclusão}

A realização deste estudo permitiu concluir que a maior parte dos idosos apresentou limiares auditivos normais ou perda auditiva de grau leve a moderado. A sintomatologia depressiva foi detectada em um número menor de indivíduos e não houve associação entre perda auditiva e sintomatologia depressiva. A idade e o sexo exerceram influência sobre a presença de perda auditiva. 


\section{A study of eldely and hearing in association with depressive simptomatology}

\section{Abstract}

Hearing loss is a major cause of social isolation in the elderly and can lead to depression. The aim of this study was to verify the existence of an association between the presence and degree of hearing loss and depressive symptoms in a group of elderly as well as to analyze the association between these two variables and the influence of age and sex. Elderly subjects were evaluated by means of pure tone audiometry with pure tone thresholds of search by air and bone conduction, for the presence, type and degree of hearing loss. The evaluation also included the application of the Geriatric Depression Scale (GDS) to observe the presence of depressive symptoms. The sample consisted of 96 elderly, $74(77.1 \%)$ were female, aged 61-90 years (mean 72.1 \pm 7.2 years). We noticed the presence of hearing loss in $64(66.7 \%)$ elderly, with a prevalence of mild and moderate hearing loss. Regarding depressive symptoms, this was observed in $22(22.9 \%)$ elderly. No association was observed between the presence and degree of hearing loss and depressive symptomatology. Was no association between hearing loss and age. It was concluded that most of the elderly had some hearing loss from mild to moderate. Depressive symptoms were detected in a small number of individuals and there was no association between hearing loss and depressive symptomatology. The age have any influence on hearing loss. There was no association between sex, hearing loss and depressive symptomatology.

Keywords: Hearing loss. Depression. Aging.

\section{Referências}

AMARAL, L. C. G.; SENA, A. P. R. C. Perfil Audiológico dos pacientes da terceira idade atendidos no Núcleo de Atenção Médica Integrada da Universidade de Fortaleza. Fono Atual, São Paulo, v. 27, n. 7, p. 58-64, 2004.

BARALDI, G. S.; ALMEIDA, L. C.; BORGES, A.C. Evolução da perda auditiva no decorrer do envelhecimento. Revista Brasileira de Otorrinolaringologia, São Paulo, v. 73, n. 1, p. 64-70, jan/fev. 2007.

BÉRIA, J. U. et al. Hearing impairment and socioeconomic factors: a populationbased survey of na urban locality in southern Brazil. Revista Panamericana de Salud Publica, Washington, v. 21, n. 6, p. 381-387, June 2007.

BEVILACQUA, M. C. et al. Survey of hearing disorders in na urban population in Rondonia, Northern Brazil. Revista de Saúde Pública, São Paulo, v. 47, n. 2, p. 309-315, abr. 2013.

BESS, F. H.; WILLIAMS, A. H.; LICHTENSTEIN, M. J. Avaliação audiológica dos idosos. In: MUSIEK, F. E.; RINTELMANN, W. F. Perspectivas atuais em avaliação auditiva. São Paulo: Manole; 2001. p. 343-369.

BILTON, T. et al. Prevalência da deficiência auditiva em uma população idosa. O Mundo da Saúde, São Paulo, v. 21, n. 4, p. 218-225, 1997.

BLUMENTHAL, J. A. et al. Effects of exercise Training on older patients with major depression. Archives of Internal Medicine, Chicago, v. 159, n. 19, p. 2349-2356, Oct. 1999.

CHOU, K. L.; CHI, I. Combined effect of vision and hearing impairment on depression in elderly Chinese. International Journal of Geriatric Psychiatry, Malden/USA v. 19, n. 9, p. 825-832, Sept. 2004. 
LI, C. M. et al. Hearing impairment associated with depression in US adults, National Health and Nutrition Examination Survey 2005-2010. JAMA Otolaryngology Head and Neck Surgery, Chicago, v. 140, n. 4, p. 293-302, 2014.

COELHO, C. L. S. et al. Higher prevalence of major depressive symptoms in Brazilians aged 14 and older. Revista Brasileira de Psiquiatria, São Paulo, v. 35, n. 2, p. 142-149, abr./jun. 2013.

COSTA. E. et al. Agreement between the Geriatric Depression Scale and the General Health Questionnarie in a population-based elderly cohort: The Bambuí Health \& Agein Study. Clinical Gerontologist, New York, v. 26, n. 3/4, p. 69-82, 2003.

CRUICKSHANKS, K. J. et al. The 5-Year Incidence and progression of Hearing Loss. Archives of Otorhinolaryngology. Head and Neck Surgery, Chicago, v. 129, n. 10, p. 1041-1046, Oct. 2003.

DANIELEWICZ, A. L.; BARBOSA, A. R.; DEL DUCA, G. F. O estado nutricional, desempenho físico e capacidade functional de uma população de idosos no sul do Brasil. Revista da Associação Médica Brasileira, São Paulo, v. 60, n. 3, p. 242-248, 2014.

DESLANDES, A. The biological clock keeps ticking, but exercise may turn it back. $A r$ quivos de Neuropsiquiatria, São Paulo, v. 71, n. 2, p. 113-118, Feb. 2013.

DUNN, A. L. et al. Exercise Treatment for Depression: Efficacy and Dose Response. American Journal of Preventive Medicine, $\mathrm{v}$. 28 , n. 1, p. 1-8, 2005.

FARIA, A. C. B. N.; BARRETO, S. M.; PASSOS, V. M. A. Sintomatologia depressiva em idosos de um plano de saúde. Revista Médica de Minas Gerais, Belo Horizonte, v. 18, n. 3, p. 175-182, jul./set. 2008.

FERREIRA, P. C. S.; TAVARES, D. M. S. Prevalência e fatores associados ao indicativo de depressão entre idosos residentes na zona rural. Revista da Escola de Enfermagem da USP, São Paulo, v. 47, n. 2, p. 401-407, abr. 2013.
FIGUEIREDO, A. C. et al. Depressão no idoso. In: TERRA, N. L.; DORNELLES, B. Envelhecimento bem-sucedido. Porto Alegre: EDIPUCRS; 2002. p. 149-152.

GARDNER, M.; SLENKOVICH, N. C. Disacusia neurossensorial. In: JAFEK, B.W., MURROW, B.W. Segredos em Otorrinolaringologia. Porto Alegre: Artmed, 2006.

GRECO, M. C., RUSSO, I. C. P. Achados audiológicos em indivíduos idosos atendidos em uma clínica particular de São Paulo-SP. Revista Acta AWHO, São Paulo, v. 24, n. 4, p. 245-254. 2006.

INSTITUTO BRASILEIRO DE GEOGRAFIA E ESTATÍSTICA - IBGE. Censo Demográfico 2010: características gerais da população, religião e pessoas com deficiência. Disponível em: <ftp://ftp.ibge.gov.br/Censos/Censo_Demografico_2010/Caracteristicas_Gerais_Religiao_Deficiencia/tab1_1.pdf $>$. Acesso em: 20 nov. 2012.

KIDD, A.R.; BAO, J. Recent advances in the study of age-related hearing loss- a minireview. Gerontology, Austria, v. 58, n. 6, p. 490-496, June 2012.

KIM, T.S., CHUNG, J.W. Evaluation of age-related hearing loss. Korean Journal of Audiology, Seoul/South Korea, v. 17, n. 2, p. 50-53, Sept. 2013.

LEITE, V. M. M. et al. Depressão e envelhecimento: estudo nos participantes do Programa Universidade Aberta da Terceira Idade. Revista Brasileira de Saúde Materno Infantil, Recife, v. 6, n. 1, p. 31-38, jan./mar. 2006.

MACIEL, A. C. G.; GUERRA, R. O. Prevalência e fatores associados à sintomatologia depressiva em idosos residentes no Nordeste do Brasil. Jornal Brasileiro de Psiquiatria, Rio de Janeiro, v. 55, n. 1, p. 26-33, jan./mar. 2006.

MALHOTRA, R.; CHAN, A.; OSTBYE, T. Prevalence and correlates of clinically significant depressive symptoms among elderly people in Sri-Lanka: findings a national survey. International Psychogeriatrics, Cambridge, v. 22, n. 2, p. 227-237, Mar. 2010. 
MATTOS, L. C.; VERAS, R. P. A prevalência da perda auditiva em uma população de idosos da cidade do Rio de Janeiro: um estudo seccional. Revista Brasileira de Otorrinolaringologia, São Paulo, v. 73, n. 5, p. 654-659, ago./out. 2007.

McCORMACK, A.; FORTNUM, H. Why do people fitted with hearing aids not wear them? International Journal of Audiology, London, v. 52, n. 5, p. 360-368, May 2013.

McDONALL, M. C. The effects of developing a dual sensory loss on depression in older adults: a longitudinal study. Journal of Aging and Health, Galveston, v. 21, n. 8, p. 1179-1199, Dec. 2009.

MENESES, C. et al. Prevalência de perda auditiva e fatores associados na população idosa de Londrina, Paraná: estudo preliminar. Revista CEFAC, São Paulo, v. 12, n. 3, p. 384-392, maio/jun. 2010.

MILLÁN-CALENTI, J. C. et al. Relación entre el deficit sensorial auditivo y depresión en personas mayores: revisión de la literatura. Revista Española de Geriatría y Gerontología, v. 46, n. 1, p. 30-33, 2011.

MINGHELLI, B. et al. Comparação dos níveis de ansiedade e depressão entre idosos ativos e sedentários. Revista de Psiquiatria Clínica, São Paulo, v. 40, n. 2, p. 71-76, 2013.

MONZANI, D. et al. Psychological profile and social behaviour of working adults with mild to moderate hearing loss. Acta Otorhinolaryngologica Italian, v. 28, n. 2, p. 61-66, Apr. 2008.

NACHTEGAAL, J. et al. The association between hearing status and psychosocial health before the age of 70 years: results from an internet-based national surveyon hearing. Ear and Hearing, Philadelphia, v. 30, n. 3, p. 302-312, June 2009.

NÓBREGA, J. D.; CÂMARA, M. F. S.; BORGES, A. C. C. Audição do idoso: análise da percepção do prejuízo auditivo, perfil audiológico e suas correlações. Revista Brasileira em Promoção de Saúde, Fortaleza, v. 21, n. 1, p. 39-46, 2008.
ORGANIZAÇÃO MUNDIAL DA SAÚDE. WHO/PDH/97.3 Geneva: WHO, 1997.

PEDALINI, M. E. B., et al. A análise do perfil audiológico de idosos através de testes da função auditiva periférica e central. $R e$ vista Brasileira de Otorrinolaringologia, São Paulo, v. 63, n. 5, p. 489-496, set./out. 1997.

ROSENHALL, U. Ageing in the auditory and vestibular systems. In: LUXON, L. Textbook of Audiological Medicine - clinical aspects of hearing and balance. London: Martin Dunitz, 2003.

SAMELLI, A. G. et al. Comparação entre avaliação audiológica e screening: um estudo sobre presbiacusia. Brazilian Journal of Otorhinolaryngology, São Paulo, v. 77, n. 1, p. 70-76, jan./fev. 2011.

SHEIKH, J. I.; YESAVAGE, J. A. Geriatric depression scale (GDS): recent evidence and development of a shorter version. Clinical Gerontology, Cambridge, v. 5, n. 1/2, p. 165173, jun. 1986.

SILVA, B. S. R. et al. Caracterização das queixas, tipo de perda auditiva e tratamento de indivíduos idosos atendidos em uma clínica particular de Belém-PA. Arquivos Internacionais de Otorrinolaringologia, São Paulo, v. 11, n. 4, p. 387-395, 2007.

TEIXEIRA, A. R. et al. Associação entre Perda Auditiva e Sintomatologia Depressiva em Idosos. Arquivos Internacionais de Otorrinolaringologia, São Paulo, v. 14, n. 4, p. 444-449, out./dez. 2010.

TENÓRIO, J. P. et al. Comparação entre critérios de classificação dos achados audiométricos em idosos. Jornal da Sociedade Brasileira de Fonoaudiologia, São Paulo, v. 23, n. 2, p. 114-118, 2011.

VALETE-ROSALINO, C. M.; ROSENFELD, S. Triagem auditiva em idosos: comparação entre auto-relato e audiometria. Revista Brasileira de Otorrinolaringologia, São Paulo, v. 71, n. 2, p. 193-200, mar./abr. 2005. 
VERAS, R. Envelhecimento populacional contemporâneo: demandas, desafios, inovações. Revista de Saúde Pública, São Paulo, v. 43, n. 3, p. 548-554, maio/jun. 2009.

ZAITUNE, M. P. A. et al. Fatores associados à prática de atividade física global e de lazer em idosos: Inquérito de saúde no estado de São Paulo (ISA-SP), Brasil. Cadernos de Saúde Pública, São Paulo, v. 26, n. 8, p. 1606-1618, ago. 2010 . 